\title{
Erratum to: A control-orientated analytical model for a cyclorotor wave energy device with $\mathrm{N}$ hydrofoils
}

\author{
Andrei Ermakov $^{1}$ (D) John V. Ringwood ${ }^{1}$
}

Received: 5 July 2021 / Accepted: 9 July 2021 / Published online: 6 August 2021

(c) Springer Nature Switzerland AG 2021

\section{Erratum to:}

Journal of Ocean Engineering and Marine Energy (2021)

7:201-210

https://doi.org/10.1007/s40722-021-00198-8

After the publication of the original article, it was discovered that there was a typing error in Eq. (12).

The published version of Eq. (12) is:

$$
\begin{aligned}
\mathcal{F}(z, t)= & \frac{\Gamma(t)}{2 \pi \dot{\mathrm{i}}} \log \left[\frac{z-c(t)}{z-\tilde{c}(t)}\right] \\
& +\frac{\sqrt{g}}{\pi \dot{\mathrm{i}}} \int_{0}^{t} \Gamma(\tau) D\left[\frac{\sqrt{g}(t-\tau)}{2 \sqrt{\dot{\dot{I}}(z-\tilde{c}(\tau))}}\right] \mathrm{d} \tau
\end{aligned}
$$

The correct version of Eq. (12) is:

$$
\begin{aligned}
\mathcal{F}(z, t)= & \frac{\Gamma(t)}{2 \pi \dot{\mathrm{I}}} \log \left[\frac{z-c(t)}{z-\tilde{c}(t)}\right] \\
& -\frac{2 \dot{\mathrm{i}} \sqrt{g}}{\pi} \int_{0}^{t} \frac{\Gamma(\tau)}{\sqrt{\dot{\mathrm{i}}(z-\tilde{c}(\tau))}} D\left[\frac{\sqrt{g}(t-\tau)}{2 \sqrt{\dot{\mathrm{i}}(z-\tilde{c}(\tau))}}\right] \mathrm{d} \tau
\end{aligned}
$$

However, we do not reference this formula in the original article text and do not use it for validation of our model against the Atargis results. It is just an intermediate step in the derivation of the final Eqs. (16)-(21).

Authors also recommend to use more accurate new equations for components of the wake velocity field $\mathbf{V}_{\mathbf{H W}}$ (Eqs. 18, 19):

This project has received funding from the European Union's Horizon 2020 research and innovation programme under Grant agreement no. 851885 .

The original article can be found online at https://doi.org/10.1007/ s40722-021-00198-8.

\section{Andrei Ermakov}

ermakovandreim@gmail.com

1 Centre for Ocean Energy Research, Maynooth University, Maynooth, Co. Kildare, Ireland

$$
\begin{aligned}
& \left(V_{\mathrm{HW}}\right)_{x}=\operatorname{Re}\left[\int_{0}^{t} \frac{\Gamma_{i}[\tau] g(t-\tau)}{2 \pi\left(x_{j}-x_{i}[\tau]+\dot{\mathrm{i}}\left(y_{j}+y_{i}[\tau]\right)\right)^{2}}\right. \\
& \times\left(1+\frac{2 \dot{\mathbf{I}}\left(\left(x_{j}-x_{i}[\tau]\right)+\dot{\mathbf{I}}\left(y_{j}+y_{i}[\tau]\right)\right)-g(t-\tau)^{2}}{\sqrt{g}(t-\tau) \sqrt{\dot{\mathbf{I}}\left(x_{j}-x_{i}[\tau]+\dot{\mathbf{I}}\left(y_{j}+y_{i}[\tau]\right)\right)}}\right. \\
& \left.\left.\cdot D\left[\frac{\sqrt{g}(t-\tau)}{2 \sqrt{\dot{i}\left(x_{j}-x_{i}[\tau]+\dot{i}\left(y_{j}+y_{i}[\tau]\right)\right)}}\right]\right) \mathrm{d} \tau\right] \\
& \left(V_{\mathrm{HW}}\right)_{y}=-\operatorname{Im}\left[\int_{0}^{t} \frac{\Gamma_{i}[\tau] g(t-\tau)}{2 \pi\left(x_{j}-x_{i}[\tau]+\dot{\mathrm{i}}\left(y_{j}+y_{i}[\tau]\right)\right)^{2}}\right. \\
& \times\left(1+\frac{2 \dot{\mathbf{I}}\left(\left(x_{j}-x_{i}[\tau]\right)+\dot{\mathbf{I}}\left(y_{j}+y_{i}[\tau]\right)\right)-g(t-\tau)^{2}}{\sqrt{g}(t-\tau) \sqrt{\dot{\mathbf{I}}\left(x_{j}-x_{i}[\tau]+\dot{\mathbf{I}}\left(y_{j}+y_{i}[\tau]\right)\right)}}\right. \\
& \left.\left.\cdot D\left[\frac{\sqrt{g}(t-\tau)}{2 \sqrt{\dot{1}\left(x_{j}-x_{i}[\tau]+\dot{i}\left(y_{j}+y_{i}[\tau]\right)\right)}}\right]\right) \mathrm{d} \tau\right]
\end{aligned}
$$

Rather than original equations (Eqs. 18, 19), for which some assumptions used for separations of the real and imaginary parts may lead to calculation error up to $10 \%$ :

$$
\begin{gathered}
\left(V_{\mathrm{HW}}\right)_{x}=-\int_{0}^{t} \frac{\Gamma_{i}(\tau) \sqrt{g}}{2 \pi} \cos \left(x_{j}-x_{i}[\tau]\right)\left(-\frac{\sqrt{g}(t-\tau)}{\left(y_{j}+y_{i}[\tau]\right)^{2}}\right. \\
\left.+\frac{\left(g(t-\tau)^{2}+2\left(y_{j}+y_{i}[\tau]\right)\right)}{\left(-y_{j}-y_{i}[\tau]\right)^{5 / 2}} D\left[\frac{\sqrt{g}(t-\tau)}{2 \sqrt{-y_{j}-y_{i}[\tau]}}\right]\right) \mathrm{d} \tau \\
\left(V_{\mathrm{HW}}\right)_{y}=\int_{0}^{t} \frac{\Gamma_{i}[\tau] \sqrt{g}}{2 \pi} \sin \left(x_{j}-x_{i}[\tau]\right)\left(-\frac{\sqrt{g}(t-\tau)}{\left(y_{j}+y_{i}[\tau]\right)^{2}}\right. \\
\left.+\frac{\left(g(t-\tau)^{2}+2\left(y_{j}+y_{i}[\tau]\right)\right)}{\left(-y_{j}-y_{i}[\tau]\right)^{5 / 2}} D\left[\frac{\sqrt{g}(t-\tau)}{2 \sqrt{-y_{j}-y_{i}[\tau]}}\right]\right) \mathrm{d} \tau
\end{gathered}
$$

We express our gratitude to Dr. Casey Fagley (from the Atargis Energy Corporation Atargis (2020)), who reproduced our results and pointed out this issue to us. 


\section{Reference}

Atargis (2020) The energy corporation website. https://atargis.com//. Accessed 20 Mar 2021
Publisher's Note Springer Nature remains neutral with regard to jurisdictional claims in published maps and institutional affiliations. 